\title{
Ability profiles: A framework for conceptualizing dimensions of ability
}

\author{
Erin Scanlon and Jacquelyn J. Chini \\ Physics Department, University of Central Florida, 4111 Libra Drive, Orlando, FL, 32816
}

\begin{abstract}
Every person has abilities across a multidimensional spectrum; abilities can vary within a person across these dimensions as well as between people along the same dimensions. This paper introduces a preliminary framework for conceptualizing dimensions of ability which we call ability profiles. Our purpose in developing this framework is not to categorize other people, but rather to support research into existing structures that privilege those with strengths in particular dimensions or create a barrier for those with limitations in certain dimensions. Such an analysis is useful for developing inclusive and accessible curricular materials and practices. Through literature reviews, we have developed a framework that describes largegrain dimensions of ability: physical, visual, hearing, cognitive, health, and emotional-behavioral. This paper will introduce ability profiles and the dimensions of ability, describe the relationship between the framework and specific diagnoses, and demonstrate utility of the framework for the physics classroom.
\end{abstract}

\section{INTRODUCTION}

Courses are typically developed with the "average" student in mind; students who are similar to the "traditional" student with all of the accompanying expected knowledge, skills, experiences, culture, abilities, and interests. However, current neurological research indicates that all people vary in their needs, abilities, and interests across a multidimensional spectrum [1]. As Goodley states "the ideal able-bodied identity can never be achieved" (p. 52) [2]. Curricula designed for the "average" student are, in fact, designed for no one.

Many faculty lack the knowledge and skills to support students who do not fall in the "typical" range on the multidimensional ability spectrum [3]. Many instructors feel they do not know how to begin improving the accessibility of their courses because they do not know which students in their courses need extra or different support [4]. Also, some students do not want to, know how to, or cannot afford to disclose their disability diagnoses; diagnosis and disclosure of disability are required to access school-sanctioned accommodations. The purpose of this paper is to introduce a toy model, called ability profiles, that allows for evaluation of the accessibility of courses by highlighting expected variation along multiple dimensions relevant to engaging in a learning community, such as a physics course. This framework can help instructors investigate the accessibility of their courses without requiring students to disclose their disability diagnoses.

Our ability profiles model is intended to be used for curricular development and research to support variation in students' abilities, needs, and interests. We have previously examined popular, research-based introductory physics curricula and found many places where the curricula inherently did not support variation in students' needs, interests, and abilities [5-6]. For example, the curricula we examined did not promote expectations and beliefs about the course and physics in general that would help optimize students' motivation. We want to help instructors with the task of moving their courses toward both accessibility for students with documented impairments and more broadly toward more fully supporting all students.

\section{MODEL DEVELOPMENT}

Three principles guided the development of the ability profiles model: 1) the model must be understandable by and useful for the physics community; 2) the model should support curriculum development and research; and 3) the dimensions of ability should be at a similar grain-size. We followed the principles in this order, meaning we valued creating dimensions that could be understood by a typical physics instructor over maintaining identical grain-size.

To develop the model, we searched the literature for types of disabilities with the idea that if a person can be disabled in a category, then they can also be abled in that same category; as Goodley explains, culturally we are "incapable, often, of being able to define what we mean by a normal or able body, we are more adept at describing an abnormal body" (pg. 15) [2]. We searched literature from a myriad of disciplines, including disability studies [7], education [8], medicine [9], social science [10], psychology [11], technology [12], and governmental organizations [13] with both domestic [14] and international [15] origins to ensure inclusion of a variety of conceptualizations of (dis)ability in the model. Existing frameworks for categorizing (dis)ability have focused on the specifics of the population in the study [8], defined categories based on specific diagnoses [16] or made definitions as a means of determining who gets access and services [13]. These types of definitions do not lend themselves to the examination of the accessibility of curricula because they either are not broad enough to encompass the full range of abilities or they have been previously used as a means to exclude individuals from the postsecondary environment. 
We combined similar literature-based emergent categories of ability to form six dimensions of ability: physical, health, cognitive, visual, hearing, and emotionalbehavioral. The only emergent category that did not fit in with these six dimensions is related to a person's ability for self-care and independent living; this was not included in our dimensions as it had high collinearity with the other dimensions.

Next, each dimension was defined using results of another literature review on the varying definitions of each type of (dis)ability [17-22]. Finally, the toy model was discussed with disability experts to ensure that the model is consistent with current perspectives from that discipline.

\section{ABILITY PROFILES}

Through this process we have developed a toy model, called ability profiles, which describes dimensions of ability along which people can vary. The model does not take into account connections between the dimensions. For example, executive function skills (e.g., working memory, flexible thinking, self-control - which fall under the cognitive dimension in this model) can mediate the efficacy of abilities along the sensory dimensions (physical, health, visual, hearing) because if a person cannot regulate the use of abilities along different dimensions then those abilities are not as useful.

Below, we describe each dimension and discuss sample diagnoses that would likely affect a person's ability along the dimension. However, this model does not only relate to disabilities and diagnoses; all people fall on a spectrum of ability along each dimension. We list a few sample diagnoses to give more concrete examples.

The physical dimension of ability relates to a person's strength, mobility, dexterity, and endurance. As an example, a female student may have difficulties using a particularly large scanning tunneling microscope, despite being an expert user of a smaller model, because of her small arm span [23]. Such a tool would not support individual variation along the physical dimension. Similarly, a person diagnosed with cerebral palsy, multiple sclerosis, or who has lost a limb would likely experience limitations along this dimension of ability.

The health dimension relates to medical conditions that affect a person's life. A person with diabetes may experience fluctuations in blood sugar levels that make it dangerous to sit through a three-hour long class without a snack. Some courses have policies that make this easier (allowing for snack breaks) or more difficult (time constrained laboratories where no food or drink is allowed) for the student. Similarly, a person diagnosed with a seizure disorder, heart disease, or food or environmental allergies would likely experience limitations along this dimension.

The cognitive dimension relates to a person's thinking, conceptualizing, planning, remembering, interpreting, understanding, metacognition, decision making, and self- management. Many instructors have likely come across a student who seems disorganized, seems to not follow through with tasks, has difficulties with time-management, or lacks motivation [24]. This person likely has difficulties with executive function skills and would experience limitations along the cognitive dimension. Similarly, a person diagnosed with attention deficit/hyperactivity disorder, autism spectrum disorder, or intellectual disabilities would likely experience limitations along this dimension.

The visual dimension relates to visual acuity (i.e., sharpness of vision) and color vision. For example, a person with color vision deficiencies may not be able to distinguish information presented in certain color combinations common in poster presentations and conference talks and thus experiences a limitation along the visual dimension of ability [25]. Similarly, a person with hyperopia, myopia, low vision, or total blindness would have a limitation along this dimension.

The hearing dimension relates to a person's sensitivity to the loudness and the pitch of sound. For example, a person with tinnitus would experience ringing or buzzing in their ears which can make hearing the instructor's lecture or group discussions difficult. Thus, a person with tinnitus would likely experience limitations along the hearing dimension of ability. Similarly, a person who is deaf, hard of hearing, or has pitch-specific hearing loss would experience a limitation along this dimension.

The emotional-behavioral dimension relates to a person's emotions, attitudes, and behaviors. For example, a person with difficulties in or anxiety about social situations may find it difficult to work in small groups to solve physics problems and may experience limitations along the emotionalbehavioral dimension. Similarly, a person diagnosed with depression, anxiety, or obsessive-compulsive disorder would experience limitations along this dimension.

\section{FOCUSING ON THE (DISABLING) LEARNING ENVRIONMENT}

We shift our gaze from individual students to the (potentially) disabling learning environment. While the previous section described the dimensions of ability and discussed specific examples of variation along these dimensions, we intend the model to be used to describe the potentially disabling curricular materials and learning environments rather than people. Recall that instructors have reported difficulty identifying students who need specific supports or accommodations. Instead, we intend instructors, curriculum developers, and researchers to apply the framework to the curricular materials and learning environment and ask questions such as "What load does this activity put on each dimension?" and "Overall, does my course frequently place a high load on certain dimensions in a way that privileges certain abilities?"

We would like to highlight ways in which the model is NOT intended to be used. Chiefly, we are not attempting to 
place individuals along the dimensions of ability. We believe that all people, regardless of their needs, abilities, and interests, should be welcome in the physics community. We also push against the capitalist tendency to view the main role of education as preparing future workers and embrace the natural curiosity that inspires each of us to want to learn about the world [24]. As such, we do not wish this model to be used as a rationale for excluding people from physics or postsecondary education in general. This model is intended to point out the inherent variations between people along multiple dimensions of ability for use as a means of describing how the educational system currently creates barriers and affordances for different people.

\section{SAMPLE APPLICATION OF ABILITY PROFILES}

As a way of visualizing the intended use of our ability profiles model, we have created a sample radar chart that shows four types of classroom activities and the intended load that these activities place on students along the six dimensions of ability ${ }^{1}$, as shown in Fig. 1.

This radar chart is on a scale from low to high load (with medium load in the middle). We acknowledge that this type of graphic is sometimes used to display individual abilities along multiple dimensions, for example in sports or roleplaying games. We are asking the reader to instead think of our radar graph as depicting the curriculum's intended load on each dimension. We emphasize that this is the intended load because we expect that actual load will vary from student to student. For example, a student with low vision may use accommodations to access information displayed visually, shifting this load onto physical, hearing and/or emotional-behavioral dimensions. However, the depiction of high load on the visual dimension is still meaningful as it indicates the student with low vision will need to do extra work to access that information. We acknowledge that through this line of reasoning we have shifted from thinking about people (to create the dimensions) to thinking about curricula (to depict the intended load on dimensions) and back to thinking about individual students (to explain potential differences between intended and actual load). However, as we explain below, we believe that instructors and curriculum developers' work will reside in this middle step of assessing intended load.

The radar chart representation of our ability profiles model allows instructors to conduct a thought experiment to examine the places where their curricular activities are privileging some students and taxing others. If a course is composed of activities that continually have a high load along a dimension or two, then the curricula is not serving students that experience limitations along that dimension of ability.

\footnotetext{
${ }^{1}$ The curricular activity examples displayed in Fig. 2 are solely for discussion purposes. The locations of the nodes will change depending on
}
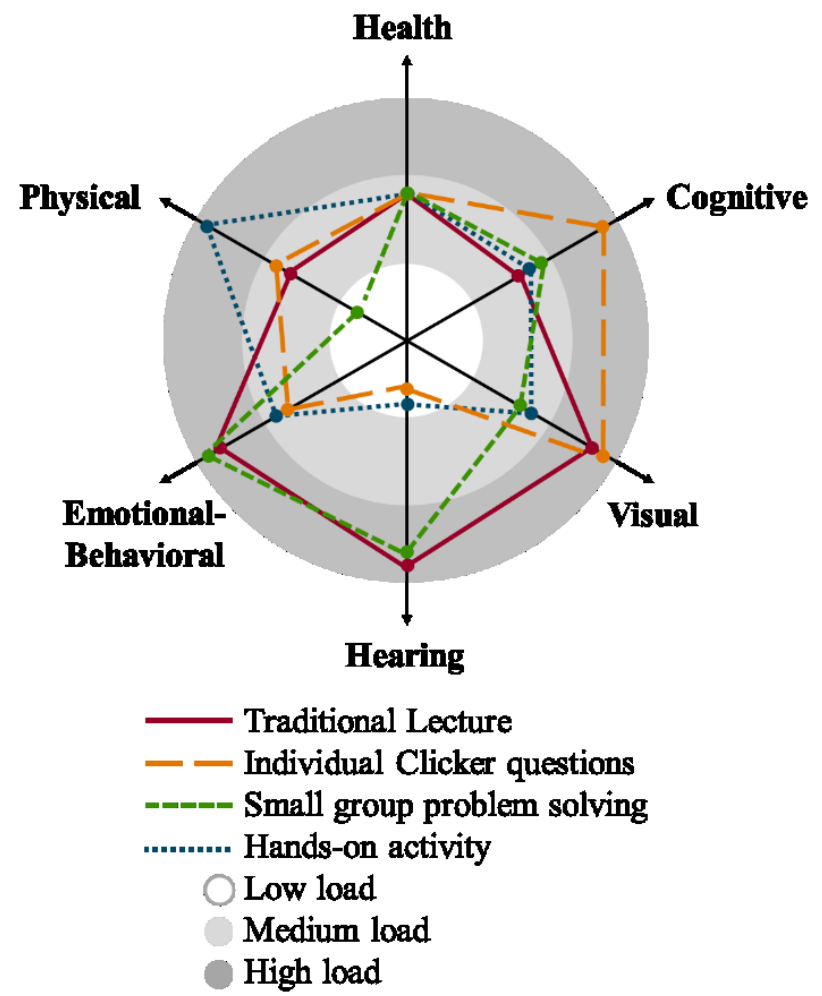

FIG. 1. Curriculum intended load on ability dimensions

As an example, the radar chart in Fig. 1 shows four popular classroom activities that are used in introductory physics courses: traditional lecture, individual clicker questions, small group problem-solving, and hands-on activities. The location of the node for each activity on each dimension of ability is qualitative in nature (ordinal data not interval). The low to high loading should be interpreted as a spectrum. Traditional lecture loads high on the hearing dimension because students are typically expected to listen to the instructor talk during the lecture time. ${ }^{1}$ Similarly, small group problem-solving loads high on the hearing dimension because students typically communicate verbally with each other during this activity. On the other hand, while working on individual clicker questions and hands-on activities students are not necessarily required to listen to another person and thus these curricular components load lower on the hearing dimension.

As another example, hands-on activities have a high load on the physical dimension of ability because students are typically required to manipulate equipment in order to participate in the activity. Clicker questions have a medium load on the physical dimension because students are tasked with clicking buttons on a clicker as a means of responding to the answer. Similarly, during a traditional lecture, students are expected to take notes during class, which is typically

the specifics of their implementation as well as the instructor, course, and institution. 
done via handwriting. Small group problem-solving, on the other hand, loads low on the physical ability dimension as students typically work in groups and only submit one written copy of their work. Thus, not every student is required to write their answers to the problems, allowing for options for those who experience limitations along the physical dimension of ability.

While using this radar chart, instructors need to take note of places where their course places a particularly high load on a certain dimension in ways that privilege those abilities. For example, if a course is composed exclusively of traditional lecture and small group problem-solving the entire course would load high on the hearing and emotionalbehavioral dimensions. This implies that students who experience limitations along these two dimensions are not being well served by this course, and conversely, students with higher ability along these dimensions are privileged. Instructors should be aware of these disparities while developing and implementing curricula.

We do not advocate for instructors to create activities or curricular components that do not tax students along all dimensions of ability (i.e., minimizing the area of each activity's loop on the radar chart) because there are some tasks that are salient to learning physics that will inherently load high along a dimension of ability. Importantly, if we create activities that have a low load on every dimension of ability, then we would not be challenging students with strengths along a particular dimension. Instead, we advocate for instructors to be aware of how their curricula tax some students and privilege others and to create options for students. Options could be provided by, for example, providing students with a choice of how to learn material (e.g., listening to lecture, discussing with classmates, working through a hands-on activity) or by rotating through activities that privilege some students and tax others with abilities that vary along the different dimensions.

\section{IMPLICATIONS AND CONCLUSION}

While access for all students is mandated by federal law [8], the next step in creating a community where all students feel welcome is balancing the needs of students with different strengths and limitations. Instructors can examine the accessibility of their curriculum by making a radar chart and critically thinking about how their curricular components privilege and tax along different dimensions of ability. Then, to close the loop, instructors can set up their classes to provide options for students to ensure their class is supporting all students. If we are not preparing for all students to be in physics courses, then we send the message that not all students are welcome in the physics community.

\section{ACKNOWLEDGEMENTS}

Thank you to Matt Marino and Eleazar Vasquez for their helpful conversations. This work is supported by NSF DUE 1612009 and NSF HRD 1750515.
[1] D. H. Rose and N. Strangman, Univ. Access Inf. Soc. 5, 381 (2007).

[2] D. Goodley Disability Studies: An Interdisciplinary Introduction $2^{\text {nd }}$ ed. (Sage Publications, London, 2017).

[3] K. Norman, D. Casseau, and G. P. Stefanich, Science Ed. 82, 2 (1998).

[4] A. Lombardi, C. Murray, and B. Dallas, J. of Post. Ed. \& Dis. 26, 221 (2013).

[5] E. Scanlon, J. Schreffler, W. James, E. Vasquez, and J. J. Chini, Phys. Rev. Phys. Ed. Res. 14, 020101 (2018).

[6] E. Scanlon, T. Legron-Rodriguez, J. Schreffler, E. Ibadlit, E. Vasquez, and J. J. Chini, Chem. Educ. Res. Pract., (2018), DOI: 10.1039/c8rp00095f

[7] B. Cobb, J. Lehmann, R. Newman-Gonchar, and M. Alwell, Career Dev. For Excep. Ind. 32 (108), 2009.

[8] S. Moisey, J. Dist. Ed. 19, 73 (2004).

[9] A. Reichard, M. Stransky, K. Phillips, M. McClain, and C. Drum, Dis. \& Health J. 10, 1039 (2017).

[10] K. A. Morris and R. J. Morris, Dis. \& Soc. 21, 613 (2006).

[11] K. Sanderson and G. Andrews, Psych. Serv. 53, 80 (2002).

[12] K. L. Crow, Tech Trends 52, 51 (2008).
[13] ADA: Americans with Disabilities Act of 1990, 42 U.S.C. $\$ 12101$ et seq. (2009).

[14] P. E. Longmuir and O. Bar-Or, Adapt. Phys. Act. Quart. 17, 40 (2000).

[15] S. M. Bindawas and V. Vennu, Int. J. Environ. Res. Pub. Health 15, 419 (2018).

[16] J. Sowers and M. Smith, J. Nurs. Ed. 43, 213 (2004).

[17] A. H. Eliassen, J. Relig. \& Health 53 (1427), 2014.

[18] R. J. Turner and S. Noh, J. Health \& Soc. Behav. 29, 23 (1988).

[19] D. B. Braddock, M. C. Rizzolo, M. Thompson, and R. Bell, J. Spec. Ed. Tech. 19, 49 (2004).

[20] P. E. Longmuir and O. Bar-Or, Adapt. Phys. Act. Quart. 17, 40 (2000).

[21] M. L. Hardman, Human Exception: Society, School, and Family (Allyn and Bacon, Boston, 1990).

[22] E. Solar, Teach. Exp. Child. 44, 40 (2011).

[23] A. J. Gonsalves, A. Danielsson, and H. Petterson, Phys. Rev. Phys. Ed. Res. 12, 020120 (2016).

[24] L. Meltzer, Promoting Executive Function in the Classroom (Guildford Publications, New York, 2010).

[25] D. De Paor, Geo. Soc. Am. Today 27, 42 (2017). 